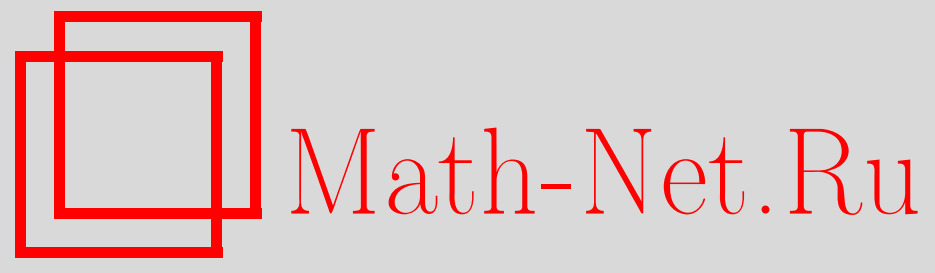

Л. А. Уварова, П. В. Лин, Моделирование процесса переноса «реакция-диффузия» в нелинейном электромагнитном поле, Вестн. Сам. гос. техн. унта. Сер. Физ.-мат. науки, 2021, номер 4, 663-675

DOI: https://doi.org/10.14498/vsgtu1869

Использование Общероссийского математического портала MathNet.Ru подразумевает, что вы прочитали и согласны с пользовательским соглашением

http://www . mathnet.ru/rus/agreement

Параметры загрузки:

IP: 52.205 .19 .152

26 апреля 2023 г., 15:09:59

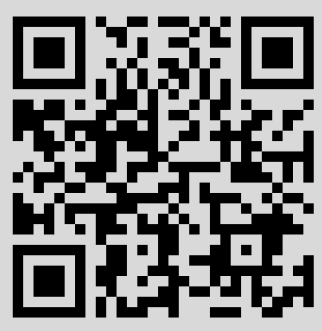


УДК 519.63:519.688

\title{
Моделирование процесса переноса «реакция-диффузия» в нелинейном электромагнитном поле
}

\author{
Л. А. Уварова, П. В. Лин
}

Московский государственный технологический университет «Станкин» Россия, 127055, Москва, Вадковский пер., 18 а.

\section{Аннотация}

Исследована модель двухкомпонентного массопереноса в неоднородной сферической системе, протекающего в нелинейном электромагнитном поле. Показано, что величины концентрации во внутренней области, на границе областей, а также концентрации, пересекшей границу и попавшей во вторую область, зависят от параметра нелинейности электродинамической задачи.

Ключевые слова: массоперенос, фазовые переход, нелинейные электродинамические уравнения, тепломассообмен.

Получение: 13 мая 2021 г. / Исправление: 30 ноября 2021 г. /

Принятие: 6 декабря 2021 г. / Публикация онлайн: 13 декабря 2021 г.

Введение. В настоящее время большой интерес представляют исследования в области фазовых переходов, барьерных эффектов и качественных преобразований гетерогенных систем (в том числе наносистем) различного типа (физических, технических, биофизических). В частности, это относится к исследованиям процессов такого рода, происходящих под воздействием электромагнитных полей, что связано с возможностью управления. Рассеяние и поглощение монохроматической волны на сферической частице, как известно, может быть описано на основе классической теории Ми [1]. Позже теория была развита в применении к цилиндрам, несферическим частицам,

\section{Научная статья}

(C) Коллектив авторов, 2021

(C) СамГТУ, 2021 (составление, дизайн, макет)

(2) () Кон Контент публикуется на условиях лицензии Creative Commons Attribution 4.0 International (https://creativecommons.org/licenses/by/4.0/deed.ru)

\section{Образец для цитирования}

Уварова Л. А., Лин П. В. Моделирование процесса переноса «реакция-диффузия» в нелинейном электромагнитном поле // Вестн. Сам. гос. техн. ун-та. Сер. Физ.мат. науки, 2021. Т. 25, № 4. C. 663-675. https://doi.org/10.14498/vsgtu1869.

\section{Сведения об авторах}

Людмила Александровна Уварова (D) https://orcid.org/0000-0003-1137-6436 доктор физико-математических наук, профессор; заведующий кафедрой; каф. прикладной математики; e-mail: uvar11@yandex.ru

Пхиъо Вэй Лин (1) https://orcid.org/0000-0002-1847-0516

аспирант; каф. прикладной математики; e-mail: phyowailinnmipt@gmail.com 
коллективам частиц. В последнее десятилетие большой интерес вызывают исследования по распространению электромагнитных волн в нелинейных средах с учетом мнимых составляющих оптических характеристик. Такие процессы могут моделироваться с помощью комплексного уравнения Гинзбурга-Ландау. Исследования такого рода были отчасти инициированы успехами в области получения точных решений нелинейных гамильтоновых уравнений в виде солитонов [2,3]. Получаемые решения имеют различные физические приложения, в частности, позволяют рассматривать эффекты, возникающие при взаимодействии электромагнитных волн с суспензиями [4]. Также в настоящее время проводятся экспериментальные и теоретические исследования в области влияния радиационной составляющей на теплоперенос в неоднородных средах, содержащих включения различных размеров [5]. При рассмотрении процессов фазовых переходов, барьерных эффектов, процессов типа «реакция-диффузия» необходимо рассматривать гетерогенные системы, которые в общем случае обладают нелинейными свойствами [6-8].

В данной работе рассматривается процесс массопереноса в гетерогенной системе, вызванный реакцией и диффузией и протекающий в нелинейном электромагнитном поле.

\section{1. Некоторые решения нелинейных электродинамических урав-} нений. В этом разделе мы рассмотрим перенос электромагнитных волн в двухслойной оптически нелинейной сфере с целью определения поглощенной электромагнитной энергии и, соответственно, источников тепла. В квазистационарном приближении система уравнений Максвелла для амплитуд электрического и магнитного векторов может быть сведена к следующим уравнениям:

$$
\begin{aligned}
& \Delta \vec{E}_{i}+k^{2} \varepsilon_{i}\left(E_{i 1}, E_{i 2}, E_{i 3}\right) \vec{E}_{i}=\nabla\left(\nabla \cdot \vec{E}_{i}\right), \\
& \Delta \vec{H}_{i}+k^{2} \varepsilon_{i}\left(E_{i 1}, E_{i 2}, E_{i 3}\right) \vec{H}_{i}=\nabla\left(k_{1 i} \times \vec{E}_{i}\right), \\
& \nabla \cdot\left(k_{1 i} \vec{E}_{i}\right)=0, \quad \nabla \cdot \vec{H}_{i}=0,
\end{aligned}
$$

где

$$
k^{2}=\frac{\omega^{2}}{c^{2}}, \quad k_{1 i}=\frac{j \omega}{c}\left(\varepsilon_{i}+j \frac{4 \pi \sigma_{i}}{c}\right), \quad j=\sqrt{-1}
$$

$E_{m i}$ - компоненты электрического вектора в ортогональной системе координат, $m=1,2,3 ; \vec{H}_{i}$ - вектор магнитного поля; индекс $i=1$ относится к внутренней области (слою) рассматриваемой сферы, а индекс $i=2-$ к внешней области. Комплексная диэлектрическая проницаемость $\varepsilon_{i}$ определяется так:

$$
\varepsilon_{i}=\varepsilon_{i}^{\prime}\left(E_{i 1}, E_{i 2}, E_{i 3}\right)+j \frac{4 \pi \sigma_{i}}{c} .
$$

Для описания нелинейной зависимости диэлектрической проницаемости от электрического вектора часто используется закон Керра или его модификация:

$$
\begin{gathered}
\varepsilon_{i}^{\prime}=\varepsilon_{i 0}-\left|\alpha_{i}\right|\left|\vec{E}_{i}\right|^{2}, \\
\varepsilon_{i}^{\prime}=\varepsilon_{i 0}-\left|\alpha_{i}\right| \vec{E}_{i}^{2},
\end{gathered}
$$


где $\alpha_{i}$ - параметр нелинейности для среды $i$. Ранее некоторые классы точных решений системы (1), (2) и (1), (3) были найдены в предположении, что среды являются подобными друг другу телами с общим центром или осью симметрии (сферы, кубы, цилиндры, торы). Особенностью этих решений является постоянство модуля электрического вектора (для зависимости (2)) или квадрата электрического вектора (для зависимости (3)). Обозначим эти решения через $\vec{E}_{i T}, \vec{H}_{i T}$. Они получены из условия $\varepsilon_{i}=0$ и, таким образом, удовлетворяют системе (1) [9, 10].

Приближенные решения вблизи этих точных решений можно найти из линеаризованных уравнений (1), представив векторы напряжений в виде

$$
\vec{E}_{i}=\vec{E}_{i T}+\vec{E}_{i}, \quad \vec{H}_{i}=\vec{H}_{i T}+\vec{H}_{i}
$$

Тогда система (1) сводится к виду [11]

$$
\begin{aligned}
& \Delta \vec{E}_{i}^{\prime}-2 k^{2}\left(\varepsilon_{i 0}+j \frac{4 \pi \sigma_{i}}{c}\right) \Delta \vec{E}_{i}^{\prime}=0, \\
& \Delta \vec{H}_{i}^{\prime}-2 k^{2}\left(\varepsilon_{i 0}+j \frac{4 \pi \sigma_{i}}{c}\right) \Delta \vec{H}_{i}^{\prime}=0, \\
& \nabla \cdot \vec{H}_{i}^{\prime}=0, \quad \nabla \cdot \vec{E}_{i}^{\prime}=0 .
\end{aligned}
$$

Граничные условия для (радиуса внутренней сферы) можно записать в виде

$$
E_{1 T}^{(t)}=E_{2 T}^{(t)}, \quad E_{1}^{\prime(t)}=E_{2}^{\prime(t)}, \quad H_{1}^{\prime(t)}=H_{2}^{\prime(t)}
$$

где индекс $t$ означает «тангенциальная составляющая».

Из уравнений (4) следует, что они формально совпадают с линейными уравнениями Гельмгольца для определения векторов напряжений. Однако роль диэлектрической проницаемости играет величина $\hat{\varepsilon}_{i}=-2 \varepsilon_{0 i}$ и роль коэффициента поглощения $-\hat{k}_{i}=8 \pi \sigma_{i} / \omega$. Будем считать, что поглощения во внешней сфере нет, т.е. $\sigma_{2}=0$. Мы также предполагаем, что справедливо следующее неравенство:

$$
R_{2} \gg R_{1}
$$

В этом случае решение для рассеянной и поглощенной электромагнитной волны $\vec{E}_{1}^{\prime}, \vec{H}_{1}^{\prime}, \vec{E}_{1}^{\prime} \vec{H}_{1}^{\prime}$ во внутренней частице совпадает с решением Ми в виде ряда с одним различием. Отличие связано с тем, что аргументом для радиальных функций является величина $\left(-j 2 \varepsilon_{20} k r\right)$, что приводит к необходимости использования функций Ганкеля второго рода (вместо функций Ганкеля первого рода), которые исчезают на бесконечности в комплексной плоскости с отрицательной мнимой частью.

2. Модель тепломассообмена. Исходя из результатов предыдущего раздела можно определить количество поглощенной электромагнитной энергии во внутренней области системы и, следовательно, величину плотности источника тепла:

$$
q=\frac{m_{1}^{\prime} m_{1}^{\prime \prime} I_{0} k\left|\vec{E}_{1}\right|^{2}}{\sqrt{\varepsilon_{20}}\left|\vec{E}_{0}\right|^{2}}
$$


где $I_{0}-$ мощность лазерного излучения, $m_{1}=m_{1}^{\prime}+j m_{1}^{\prime \prime}-$ комплексный показатель преломления,

$$
m_{1}^{\prime}=\left[\frac{1}{2}\left(\varepsilon_{10}+\left(\varepsilon_{10}^{2}+\frac{16 \pi^{2} \sigma^{2}}{\omega^{2}}\right)^{1 / 2}\right)\right]^{1 / 2}, \quad m_{1}^{\prime \prime}=\frac{2 \pi \sigma}{\omega m_{1}^{\prime}} .
$$

Конкретизируем выражение (5) для рассмотренных выше решений (для зависимости (3) как более общей). Формула получается с помощью условия (3), которое для точных решений можно записать так:

$$
\left(\vec{E}_{i T}^{\prime}+j \vec{E}_{i T}^{\prime \prime}\right)^{2}=\frac{\left(\varepsilon_{i 0}^{\prime}+j \varepsilon_{i 0}^{\prime \prime}\right)}{\left|\alpha_{1}\right|}
$$

Поскольку $\vec{E}_{T}^{\prime}$ является вектором, в зависимости от вида системы и зависимости $\vec{E}_{T}^{\prime}$ от координат могут быть получены различные зависимости для компонент данного вектора. В данном случае рассматриваются сферы и в простейшем случае зависимости компонент $E_{T r}, E_{T \theta}, E_{T \varphi}$ только от радиальной координаты решения имеют вид [12]

$$
E_{i r}=E_{i r}^{\prime}+j E_{i r}^{\prime \prime}=\left[\frac{\varepsilon_{i 0}}{2\left|\alpha_{i}\right|}+\frac{1}{2}\left(\left(\frac{\varepsilon_{i 0}}{\left|\alpha_{i}\right|}\right)^{2}+\frac{16 \pi^{2} \sigma_{i}^{2}}{\omega^{2}\left|\alpha_{i}\right|^{2}}\right)^{1 / 2}\right]^{1 / 2}, \quad E_{i \theta}=E_{i \varphi}=0 .
$$

В данном случае нас интересует внутренняя область, для которой $i=1$. Поскольку в статье рассматриваются и приближенные решения $\overrightarrow{E^{\prime}}$, необходимо также записать

$$
\vec{E}^{\prime}=\vec{E}_{1 T}^{\prime}+\operatorname{Re}\left(\vec{E}_{1}^{\prime}\right)+j\left(\vec{E}_{1 T}^{\prime}+\operatorname{Re}\left(\vec{E}_{1}^{\prime}\right)\right) .
$$

С помощью выражений (5)-(8) можно получить следующую формулу для плотности теплового потока:

$$
\begin{aligned}
q=\frac{I_{0} k m_{1}^{\prime} m_{1}^{\prime \prime}}{\sqrt{\varepsilon_{20}^{\prime}\left|E_{0}\right|^{2}}}\left[\left|E_{1}^{\prime}\right|^{2}+2\left(\operatorname{Re}\left(E_{1 r}^{\prime}\right) E_{1 r}^{\prime}+\operatorname{Im}\left(E_{1 r}^{\prime}\right) E_{1 r}^{\prime}\right)+\right. & \\
& \left.+\left(\left(\frac{\varepsilon_{i 0}}{\left|\alpha_{i}\right|}\right)^{2}+\frac{16 \pi^{2} \sigma_{i}^{2}}{\omega^{2}\left|\alpha_{i}\right|^{2}}\right)^{1 / 2}\right],
\end{aligned}
$$

где $E_{1 r}^{\prime}$ - радиальная компонента вектора $\vec{E}_{1}^{\prime}$. Здесь мы предполагаем, что внутренняя область мала (порядка нескольких нанометров). Поэтому можно усреднить плотность источника по его объему или, при необходимости, только по полярному и азимутальному углам. При усреднении по объему величина плотности источника становится постоянной $(q=\bar{q})$. Из выражений (7) и (9) можно видеть, что процедура усреднения путем интегрирования имеет смысл только для вектора $\vec{E}_{1}^{\prime}$.

Соответственно, уравнения для температуры в каждой из областей имеют следующий вид:

$$
C_{p}^{i} \rho^{(i)} \frac{\partial^{2} T(i)}{\partial t}=\chi^{(i)}\left(\frac{\partial^{2} T^{(i)}}{\partial r^{2}}+\frac{2}{r} \frac{\partial T^{(i)}}{\partial r}\right)+q_{i} f_{i}(t)
$$


где $C_{p}^{i}$ - теплоемкость при постоянном давлении, $\rho^{(i)}-$ плотность, $q_{2}=0$, $q_{1}=\bar{q}, \chi^{(i)}$ - коэффициент теплопроводности. Здесь вводится функция $f_{i}(t)$, поскольку действие источника может быть прекращено или возобновлено во внутренней области:

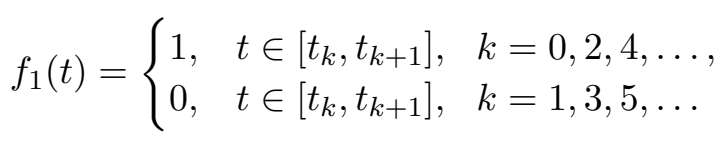

Граничные условия на внутренней границе следующие:

$$
\begin{gathered}
T^{(1)}=T^{(2)}, \quad-\chi^{(1)} \frac{\partial T^{(1)}}{\partial r}=-\chi^{(2)} \frac{\partial T^{(2)}}{\partial r}, \quad \chi=\frac{\chi^{(1)}}{\chi^{(2)}}, \\
x=\frac{r}{R_{1}}, \quad d=\frac{R_{2}}{R_{1}}, \quad b=\left(\frac{\chi C_{p}^{(1)} \rho^{(1)}}{C_{p}^{(2)} \rho^{(2)}}\right)^{1 / 2}, \quad \tau=\frac{t}{t_{\chi}}, \quad t_{\chi}=\frac{C_{p}^{(2)} \rho^{(2)} R_{1}^{2}}{\chi^{(2)}} .
\end{gathered}
$$

Решения краевой задачи (10), (11) рассматривались, например, в работе [13] при моделировании переноса электронов на большие расстояния в белковой глобуле. В нашем случае на первом этапе воздействия постоянного электромагнитного источника решение для температуры имеет вид

$$
\begin{gathered}
T^{(1)}=T_{0}^{(1)}+\frac{2 \chi \bar{q} R_{1} t_{\chi}}{C_{p}^{(1)} \rho^{(1)} r} \sum_{n=1}^{\infty} \frac{\sin \left(y_{n} b x\right) \varphi_{n} \psi_{n}(\tau)}{\sin \left(y_{n} b\right) y_{n}^{2}} \\
T^{(2)}=T_{0}^{(2)}+\frac{2 \chi \bar{q} R_{1} t_{\chi}}{C_{p}^{(1)} \rho^{(1)} r} \sum_{n=1}^{\infty} \frac{\sin \left(y_{n}(d-x)\right) \varphi_{n} \psi_{n}(\tau)}{\sin \left(y_{n}(d-1)\right) y_{n}^{2}} \\
1-\chi+\chi b y \operatorname{ctg}(b y)+y \operatorname{ctg}(y(d-1))=0 \\
\varphi_{n}=\left(1-b y_{n} \operatorname{ctg}\left(b y_{n}\right)\right)\left(1-\chi+\frac{\chi b^{2} y_{n}^{2}}{\sin ^{2}\left(b y_{n}\right)}+\frac{y_{d-1}^{2}}{\sin ^{2}\left((d-1) y_{n}\right)}\right), \\
\psi_{n}(\tau)=1-\exp \left(-y_{n}^{2} \tau\right)
\end{gathered}
$$

в течение первого периода времени воздействия источника.

Определив температуру, можно рассмотреть перенос вещества (концентрацию) в каждой из областей и между ними. Предполагается, что изменение концентрации компонентов во внутренней области происходит за счет реакции, а во внешней области - за счет диффузии:

$$
\begin{aligned}
& \frac{\partial c_{1}^{(1)}}{\partial t}=-\nu\left(T^{(1)}\right) c_{1}^{(1)} c_{2}^{(1)}, \quad \frac{\partial c_{2}^{(1)}}{\partial t}=-\nu\left(T^{(1)}\right) c_{1}^{(1)} c_{2}^{(1)} \\
& \frac{\partial c^{(2)}}{\partial t}=D\left(T^{(2)}\right) \Delta c_{1}^{(2)}, \quad c_{2}^{(2)}=0, \\
& c_{1}^{(1)}(0, r)=c_{1}^{(0)}, \quad c_{1}^{(2)}(0, r)=c_{10}, \quad c_{2}^{(1)}(0, r)=c_{20} .
\end{aligned}
$$

Здесь верхний показатель относится к среде, $D\left(T^{(2)}\right)$ - коэффициент диффузии, оператор $\Delta \equiv \frac{\partial^{2}}{\partial r^{2}}+\frac{2}{r} \frac{\partial}{\partial r}$. Скорость реакции в общем случае зависит от 
температуры по закону Аррениуса:

$$
\nu\left(T_{1}\right)=\nu_{0} \exp \left(-U / T^{(1)}\right),
$$

где $U$ - энергия, характеризующая реакцию; $R_{g}$ - универсальная газовая постоянная. В работе [14] приведены некоторые модификации данного закона, которые в конкретных случаях также могут быть использованы. Интегрируя первые два уравнения (13) вместе с выражением (14), можно рассматривать два подхода:

1) температуру, зависящую от радиальной координаты и времени;

2) среднюю температуру по объему $\bar{T}^{(1)}(t)$.

Средняя температура $\bar{T}^{(1)}(t)$ определяется по объему $4 \pi R_{1}^{3} / 3$ :

$$
\bar{T}^{(1)}=T_{0}^{(1)}+\frac{6 \psi \bar{q} t x}{c_{p}^{(1)} \rho^{1}} \sum_{n=1}^{\infty} \frac{y_{n} \varphi_{n}(\tau)}{y_{n}^{3} b}\left(\frac{1}{y_{n} b}-\operatorname{ctg}\left(y_{n} b\right)\right)
$$

На границе $R_{1}$ граничные условия имеют следующий вид:

$$
c_{1}^{(1)}=c_{1}^{(2)}, \quad \frac{\partial c_{1}^{(1)}}{\partial r}=\frac{\partial c_{1}^{(2)}}{\partial r}, \quad c_{2}^{(1)}=0 .
$$

Из системы (13) и условий (16) следует, что для определения концентрации первого компонента, проникшего во внешнюю область, в которой перенос происходит по диффузионному механизму, можно рассматривать (в зависимости от постановки задачи) как условия Дирихле, так и условия Неймана. Это возможно, так как значение $c_{1}^{(1)}$ уже будет известно. При определении этой концентрации при скорости реакции, зависящей от средней температуры (15), необходимо использовать условия Дирихле из краевых условий (16), так как условия Неймана становятся однородными (производная по координате равна нулю), и концентрация не проникает во внешнюю область.

Решение для $c_{1}^{(1)}$ имеет вид

$$
c_{1}^{(1)}=\frac{c_{1}^{0}\left(c_{20}-c_{1}^{0}\right) \exp \left(-\nu_{0}\left(c_{20}-c_{1}^{0}\right) J(t)\right)}{c_{20}-c_{1}^{0} \exp \left(-\nu_{0}\left(c_{20}-c_{1}^{0}\right) J(t)\right)}, J(t)=\int_{t_{j}}^{t} \exp \left(\frac{-U}{\bar{T}^{(1)}(S)}\right) d s,
$$

где $t_{j}$ - начало соответствующего периода времени. Решение для $c_{1}^{(2)}$ получается из третьего уравнения системы (13), первого из условий (16) и выражения (17) путем решения задачи в частных производных с неоднородными краевыми условиями Дирихле

$$
c_{1}^{(2)}\left(R_{1}\right)=c_{1}^{(1)}\left(R_{1}\right)
$$

На внешней границе можно поставить однородные условия Неймана

$$
\left.\frac{\partial c_{1}^{(2)}}{\partial r}\right|_{r=R_{2}}=0
$$


или Дирихле

$$
c_{1}^{(2)}\left(R_{2}\right)=0
$$

В этих случаях вводится величина $\xi(t)=\int D\left(\bar{T}^{(2)}\right) d t$ в качестве аналога времени. Запишем решение в случае смешанных условий как более реалистичных. Обозначим через $t=\gamma(\xi)$ функцию для определения времени, то есть обратную к функции $\xi(t)$ при заданной зависимости коэффициента диффузии от температуры. Она может быть найдена с использованием выражения для температуры во внешней области. Решение указанной задачи имеет следующий вид:

$$
\begin{aligned}
c_{1}^{(2)}=\frac{c_{1}^{(1)}(\gamma(\xi))}{r\left(R_{2}\right.}-R_{1}\left(R_{2}-r\right)^{2} & \\
& +\sum_{n=2}^{\infty} \frac{A_{n}}{r} \exp \left(-\frac{\mu_{n}^{2} \xi}{R_{2}}\right)\left(\sin \left(\frac{\mu_{n} r}{R_{2}}\right)-\operatorname{tg}\left(\frac{\mu_{n}}{d}\right) \cos \left(\frac{\mu_{n} r}{R_{2}}\right)\right) .
\end{aligned}
$$

Здесь через $\mu_{n}$ обозначены корни уравнения

$$
\mu_{n} \operatorname{tg}\left(\mu_{n}\left(1-\frac{1}{d}\right)\right)=1
$$

Поскольку рассматривается случай $d \gg 1$, решение $(15),(16)$ можно упростить:

$$
\begin{aligned}
& c_{1}^{(2)}=\frac{c_{1}^{(1)}(\gamma(\xi)) R_{1}\left(R_{2}-r\right)^{2}}{r\left(R_{2}-R_{1}\right)^{2}}+ \\
& \quad+\sum_{n=0}^{\infty} \frac{R_{2}}{r} \exp \left(-\frac{\mu_{n}^{2} \xi}{R_{2}^{2}}\right)\left(K_{1 n} \sin \left(\frac{\pi n r}{R_{2}}\right)+K_{2 n} \cos \left(\frac{\pi n r}{R_{2}}\right)\right),
\end{aligned}
$$

где

$$
\begin{gathered}
K_{10}=M_{1} \int_{\kappa}^{1} \frac{x d x}{\sin \left(\mu_{0}(1-\kappa) x\right)}-M_{2} \int_{\kappa}^{1} \frac{(1-x)^{2} d x}{\sin \left(\mu_{0}(1-\kappa) x\right)}, \\
K_{1 n}=2 M_{1} \int_{\kappa}^{1} \frac{x \cos (\pi n x) d x}{\sin \left(\mu_{0}(1-\kappa) x\right)}-2 M_{2} \int_{\kappa}^{1} \frac{(1-x)^{2} \cos (\pi n x) d x}{\sin \left(\mu_{0}(1-\kappa) x\right)}, \\
K_{2 n}=2 M_{1} \int_{\kappa}^{1} \frac{x \sin (\pi n x) d x}{\cos \left(\mu_{0}(1-\kappa) x\right)}-2 M_{2} \int_{\kappa}^{1} \frac{(1-x)^{2} \sin (\pi n x) d x}{\cos \left(\mu_{0}(1-\kappa) x\right)}, \\
M_{1}=c_{10} \cos \frac{\mu_{0} \kappa}{1-\kappa}, \quad M_{2}=c_{1}^{(0)} \kappa \cos \frac{\mu_{0} \kappa}{(1-\kappa)^{3}}, \quad \kappa=\frac{1}{d} .
\end{gathered}
$$

Величина $\mu_{0}$ определяется из уравнения $\mu_{0}=\operatorname{arctg}\left(1 / \mu_{0}\right): \mu_{0} \approx 0.863$. Интегралы вычисляются численно. 
Для дальнейшего анализа приведем некоторые частные случаи формулы для концентрации (17) с учетом (14), (15).

В первом таком случае рассмотрим изотермический процесс, который поддерживается дополнительным подводом тепла или условиями теплоизоляции после того, как произошел нагрев до некоторой температуры $\bar{T}_{k}^{(1)}=T^{(1)}\left(t_{k}\right)$, например, за первый период времени $t=t_{k}$. В этом случае для концентрации в первой области можно записать следующее выражение:

$$
c_{1}^{(1)}=\frac{c_{1}^{0}\left(c_{20}-c_{1}^{0}\right) \exp \left(-\nu_{0}\left(c_{20}-c_{1}^{0}\right) \exp \left(-\frac{U}{\bar{T}_{k}^{(1)} R_{g}}\right) t\right)}{c_{20}-c_{1}^{0} \exp \left(-\nu_{0}\left(c_{20}-c_{1}^{0}\right) \exp \left(-\frac{U}{\bar{T}_{k}^{(1)} R_{g}}\right) t\right)} .
$$

Во втором случае рассмотрим влияние изменяющейся во времени температуры на концентрацию при малых временах, когда можно ограничиться линейными членами в разложении экспоненты, входящей в функцию $\psi_{n}(t)$, в ряд, ограничиваясь несколькими первыми членами ряда $N$ (при этом коэффициенты ряда для температуры (15) убывают примерно как $1 / y_{n}^{2}$, например при $\chi \sim 1, b \sim 1$ величина $\left.y_{n}=\frac{\pi n}{d}\right)$. В этом случае выражение для интеграла $J(t)$ из (17) имеет вид

$$
\begin{aligned}
J(t) \approx \frac{T_{0}^{(1)}+\bar{q} Q t}{2.3 \bar{q} Q} & \exp \left(-\frac{U}{R_{g}\left(T_{0}^{(1)}+\bar{q} Q t\right)}\right)-\frac{T_{0}^{(1)}}{2.3 \bar{q} Q} \exp \left(-\frac{U}{R_{g} T_{0}^{(1)}}\right)+ \\
+ & \frac{U}{R_{g} \bar{q} Q}\left[\ln \left(\frac{T_{0}^{(1)}}{T_{0}^{(1)}+\bar{q} Q t}\right)+\frac{T_{0}^{(1)}}{T_{0}^{(1)}+\bar{q} Q t}+\right. \\
& \left.+\sum_{n=2}^{\infty} \frac{(-1)^{n}}{n n !}\left(\left(\frac{U}{R_{g}\left(T_{0}^{(1)}+\bar{q} Q t\right)}\right)^{n}-\left(\frac{U}{R_{g} T_{0}^{(1)}}\right)^{n}\right)\right],
\end{aligned}
$$

где

$$
Q=\frac{6 \chi}{c_{p}^{(1)} \rho^{(1)}} \sum_{n=1}^{\infty} \frac{\varphi_{n}}{y_{n}^{2} b^{2}}\left(1-y_{n} b \operatorname{ctg}\left(y_{n} b\right)\right)
$$

При получении (21) возникающий интегральный логарифм был разложен в ряд согласно известной формуле:

$$
\operatorname{li}(x)=\gamma+\ln (\ln x)+\sum_{n=1}^{\infty} \frac{(\ln x)^{n}}{n n !}
$$

где $\gamma$ - постоянная Эйлера.

Заключение. Исследована модель двухкомпонентного массопереноса в неоднородной сферической системе, протекающего в нелинейном электромагнитном поле. Из полученных решений для источника тепла, температуры и концентрации (формулы (9), (15), (17), (19), (21)) следует, что величины концентрации во внутренней области, на границе областей, а также концентрации, пересекшей границу и попавшей во вторую область, зависят от параметра нелинейности электродинамической задачи, от которого зависит плотность теплового источника. Действительно, как видно из (7), (9), в выражение 
для теплового источника входит параметр нелинейности $\alpha$. Соответственно, от него зависит температура в каждой из областей, поскольку она пропорциональна плотности теплового источника. Следовательно, через скорость реакции массоперенос зависит от характера зависимости диэлектрической проницаемости от поля. Такого рода влияние будет также иметь место и для модификаций закона Аррениуса [14]. В статье также в общем виде учитывается зависимость коэффициента диффузии от температуры. Соответственно, из формул (9), (12), (17) (18) следует, что на диффузионный механизм переноса концентрации также может влиять параметр электродинамической нелинейности. Реакция может протекать как со скоростью, практически не зависящей от температуры, так и в режиме активации, когда температура значительно влияет на скорость реакции. В данном случае в активационном режиме скорость реакции зависит от температуры внутренней области (как отмечено выше, данная температура напрямую зависит от плотности источника тепла) и радиуса $R_{1}$. В рассматриваемой задаче возможны два механизма перехода из одного режима протекания в другой. Первый механизм обусловлен варьированием параметра нелинейности. Из (7), (9), (15), (17), (20), (21) следует, что при относительно больших $\alpha$ скорость реакции и массоперенос заметно зависят от температуры, т.е. реализуется активационный режим. При малых $\alpha$ величины точных решений для электрического вектора $\vec{E}_{T}$ значительно возрастают (7). Тогда можно приближенно записать $\bar{q} \approx \frac{S}{|\alpha|}$ и соответственно $\bar{T}^{(1)} \sim \frac{S}{|\alpha|}$. Здесь через $S$ обозначена величина, приближенно полученная из (7), (9) при $\alpha \rightarrow 0$.

Отметим, что приведенный результат $\bar{q} \sim \frac{1}{|\alpha|}, \bar{T} \sim \frac{1}{|\alpha|}$ является достаточно общим и справедлив для точных решений рассматриваемого класса, опирающихся на выражение (6) и приведенных в [12]. Таким образом, происходит качественное изменение процесса переноса концентрации $c_{1}^{(1)}(t)$, обусловленное значительным возрастанием температуры в формуле $(14)$ - независимо от параметров, входящих в формулу (15), и величины энергетического барьера $U$, экспоненциальная зависимость для концентрации в (17) и (20) принимает вид $\exp \left(-\nu_{0}\left(c_{2}^{0}-c_{1}^{0}\right) t\right)$. Таким образом, происходит переход в режим, когда скорость реакции практически не зависит от температуры (в пределе $\alpha \rightarrow 0$ данный результат получается точно). В этом режиме массоперенос происходит наиболее быстро. Полагая, что $99 \%$ от скорости реакции, не зависящей от температуры, можно считать скоростью при отсутствии барьера $U$, из закона Аррениуса можно получить, что температура при этом должна быть приближенно равна $T^{+} \approx 99.602 U / R_{g}$. Соответствующую плотность теплового источника можно оценить по формуле

$$
\bar{q}^{+} \approx \frac{99.602 U}{S_{1} R_{g}}-\frac{-T_{0}^{(1)}}{S_{1}}
$$

где

$$
S_{1}=\frac{6 \chi t_{\chi}}{c_{p}^{(1)} \rho^{(1)}} \sum_{n=1}^{\infty} \frac{\psi_{n}\left(\tau_{k}\right) \varphi_{n}}{y_{n}^{4} b^{2}}\left(1-y_{n} b \operatorname{ctg}\left(y_{n} b\right)\right) .
$$

Важным также представляется рассмотрение перехода концентрации через границу раздела. Поскольку среда внешней области является непоглоща- 
ющей, скорость реакции, которая способствует распространению концентрации из области (1) в область (2), зависит от плотности теплового источника $\bar{q}$. Как показано выше, параметр нелинейности непосредственно влияет на эту величину и через температуру - на скорость реакции (12), (17), (19). Большие значения параметра $\alpha$ приводят к увеличению энергетического барьера. Это, в свою очередь, приводит к тому, что основная часть концентрации $c_{1}^{(2)}$ некоторое время находится вблизи границы раздела (возникает барьерный эффект для распространения концентрации), а затем она постепенно диффундирует далее. Такой концентрационный барьер, в свою очередь, влияет и на электродинамические характеристики внешней области. При уменьшении $\alpha$ во внешней области (так же, как и во внутренней) происходит переход от активационного к независящему от температуры режиму реакции.

Другим механизмом, переводящим активационный режим реакции в режим, который не зависит от температуры, является возникновение электромагнитного резонанса. Резонанс возникает при равенстве нулю знаменателей коэффициентов в решении $\vec{E}_{1}^{\prime}$ (решение представляет собой ряд, знаменатели членов которого зависят от $R_{1}$ и $\left.m_{1}\right)$. При этом также происходит качественное изменение зависимости $c_{1}^{(1)}(t)$ вследствие значительного возрастания температуры в выражении (17). Как и в первом рассмотренном механизме, экспоненциальная зависимость для указанной концентрации принимает вид $\exp \left(-\nu_{0}\left(c_{2}^{0}-c_{1}^{0}\right) t\right)$. Из вышеизложенного следует, что параметр электродинамической нелинейности и размер внутренней области $R_{1}$ могут являться управляющими параметрами для массопереноса в рассматриваемой системе. В том числе, от их значений может зависеть режим, в котором происходит распространение концентрации.

Конкурирующие интересы. Конкурирующих интересов не имеем.

Авторский вклад и ответственность. Все авторы принимали участие в разработке концепции статьи; все авторы сделали эквивалентный вклад в подготовку публикации. Авторы несут полную ответственность за предоставление окончательной рукописи в печать. Окончательная версия рукописи была одобрена всеми авторами.

Финансирование. Работа выполнена при поддержке Российского научного фонда (грант № 18-11-00247).

\section{Библиографический список}

1. Born M., Bhatia A. B., Wolf E. Principles of Optics: Electromagnetic Theory of Propagation, Interference and Diffraction of Light. Oxford: Pergamon Press, 1965. xxviii+808 pp.

2. Skarka V., Aleksić N., Krolikowski W., Christodoulides D., Aleksić B., Belić M. Linear modulational stability analysis of Ginzburg-Landau dissipative vortices // Opt. Quant. Electron., 2016. vol. 48, no. 4, 240. https://doi.org/10.1007/s11082-016-0514-1.

3. Aleksić B. N., Aleksić N. B., Skarka V., Belić M. Stability and nesting of dissipative vortex solitons with high vorticity// Phys. Rev. A, 2015. vol. 91, no. 4, 043832. https://doi.org/ 10.1103/PhysRevA.91.043832.

4. Skarka V., Aleksić N. B., Krolikowski W., Christodoulides D., Rakotoarimalala S., Aleksić B. N., Belić M. Self-structuring of stable dissipative breathing vortex solitons in a colloidal nanosuspension // Optics Express, 2017. vol. 25, no. 9. pp. 10090-10102. https://doi . org/10.1364/OE.25.010090.

5. Половников В. Ю. Влияние радиационного теплообмена на интенсификацию теплопереноса в тонкопленочной тепловой изоляции // Известия Томского политехнического 
университета. Инжиниринг георесурсов, 2020. Т. 331, № 8. C. 34-39. https://doi.org/ 10.18799/24131830/2020/8/2766.

6. Ильина Е. А., Сараев Л. А. Моделирование фазовых превращений и сверхупругого упрочнения нестабильных материалов // Вестн. Сам. гос. техн. ун-та. Сер. Физ.мат. науки, 2018. Т. 22, №3. C. 407-429. https://doi.org/10.14498/vsgtu1626.

7. Drin Ya., Ushenko Yu., Drin I., Drin S. About the approximate solutions to linear and non-linear pseudodifferential reaction diffusion equations // Mohyla Math. J., 2019. vol. 2. pp. 41-45. https://doi.org/10.18523/2617-70802201941-45.

8. Kuttler C. Reaction-diffusion equations with applications: Lecture Notes; Munich Technical University, 2011. https://www-m6.ma.tum.de/ kuttler/script_reaktdiff.pdf.

9. Uvarova L. A., Krivenko I. V., Ivannikov A. F. Peculiarities of stochastic resonance in disperse systems / Australian Institute of Physics 17th National Conference 2006: Refereed Papers (3-8 December, 2006; Brisbane, Australia). Canberra, 2006. 274.

10. Uvarova L. A., Babarin S. S. The movement of molecules and nanoparticles in potential field with the Casimir force in nano volumes with different optical boundaries // Physica Scripta, 2014. vol.2014, no.T162, 014053. https://doi.org/10.1088/0031-8949/2014/ T162/014053.

11. Уварова Л. А., Федянин В. К. Рассеяние электромагнитной волны на сферической частице с нелинейными свойствами: Препринт № Р17-89-372. Дубна: ОИЯИ, 1989. $7 \mathrm{c.}$

12. Уварова Л. А. Некоторые точные решения для вектора напряженности электрического поля в сопряженных нелинейных средах: Препринт № P17-87-693. Дубна: ОИЯИ, 1987. $14 \mathrm{c}$.

13. Лахно В. Д., Уварова Л. А. Влияние электронного разогрева белков на скорость электронного транспорта: Препринт. Пущино: Пущинский научный центр РАН, 1993. 13 с.

14. Чикова О. А. О структурных переходах в сложнолегированных расплавах // Известия высших учебных заведений. Черная металлургия, 2020. Т. 63, № 3-4. С. 261-270. https://doi .org/10.17073/0368-0797-2020-3-4-261-270. 


\title{
MSC: 35Q60
}

\section{Modeling of the "reaction-diffusion" transfer process in a nonlinear electromagnetic field}

\author{
L. A. Uvarova, P. W. Linn \\ Moscow State Technological University (Stankin) \\ 18 a, Vadkovsky lane, Moscow, 127055, Russian Federation.
}

\begin{abstract}
The paper deals with a model of two-component mass transfer in an inhomogeneous spherical system. A model of two-component mass transfer in an inhomogeneous spherical system occurring in a nonlinear electromagnetic field is investigated. It is shown that the concentration in the inner region, at the boundary of the regions, as well as the concentration that crossed the border and got into the second region, depend on the nonlinearity parameter of the electrodynamic problem.
\end{abstract}

Keywords: mass transfer, phase transition, nonlinear electrodynamic equations, heat and mass transfer.

Received: $13^{\text {th }}$ May, 2021 / Revised: $30^{\text {th }}$ November, $2021 /$

Accepted: $6^{\text {th }}$ December, $2021 /$ First online: $13^{\text {th }}$ December, 2021

Competing interests. The authors declare no conflicts of interests.

Authors' contributions and responsibilities. Each author has participated in the article concept development; the authors contributed equally to this article. The authors are absolutely responsible for submit the final manuscript to print. Each author has approved the final version of manuscript.

Funding. This work was supported by the Russian Science Foundation (grant no. 1811-00247).

\section{Research Article}

(C) Authors, 2021

(C) Samara State Technical University, 2021 (Compilation, Design, and Layout)

(우) The content is published under the terms of the Creative Commons Attribution 4.0 International License (http://creativecommons.org/licenses/by/4.0/)

Please cite this paper in press as:

Uvarova L. A., Linn P. W. Modeling of the "reaction-diffusion" transfer process in a nonlinear electromagnetic field, Vestn. Samar. Gos. Tekhn. Univ., Ser. Fiz.-Mat. Nauki [J. Samara State Tech. Univ., Ser. Phys. Math. Sci.], 2021, vol. 25, no. 4, pp. 663-675. https://doi.org/10.14498/vsgtu1869 (In Russian).

\section{Authors' Details:}

Lyudmila A. Uvarova (1) https://orcid.org/0000-0003-1137-6436

Dr. Phys. \& Math. Sci., Professor; Head of Dept.; Dept. of Applied Mathematics;

e-mail: uvar11@yandex.ru

Phyo Wai Linn (1) https://orcid.org/0000-0002-1847-0516

Postgraduate Student; Dept. of Applied Mathematics; e-mail: phyowailinnmipt@gmail.com 


\section{References}

1. Born M., Bhatia A. B., Wolf E. Principles of Optics: Electromagnetic Theory of Propagation, Interference and Diffraction of Light. Oxford, Pergamon Press, 1965, xxviii +808 pp.

2. Skarka V., Aleksić N., Krolikowski W., Christodoulides D., Aleksić B., Belić M. Linear modulational stability analysis of Ginzburg-Landau dissipative vortices, Opt. Quant. Electron., 2016, vol. 48, no. 4, 240. https://doi.org/10.1007/s11082-016-0514-1.

3. Aleksić B. N., Aleksić N. B., Skarka V., Belić M. Stability and nesting of dissipative vortex solitons with high vorticity, Phys. Rev. A, 2015, vol.91, no.4, 043832. https://doi.org/ 10.1103/PhysRevA.91.043832.

4. Skarka V., Aleksić N. B., Krolikowski W., Christodoulides D., Rakotoarimalala S., Aleksić B. N., Belić M. Self-structuring of stable dissipative breathing vortex solitons in a colloidal nanosuspension, Optics Express, 2017, vol. 25, no. 9, pp. 10090-10102. https://doi. org/10.1364/OE.25.010090.

5. Polovnikov V. Yu. Influence of radiation heat exchange on the intensification of heat transfer in thin-film thermal insulation, Bulletin of the Tomsk Polytechnic University. Geo Assets Engineering, 2020, vol.331, no. 8, pp. 34-39 (In Russian). https://doi.org/ $10.18799 / 24131830 / 2020 / 8 / 2766$.

6. Ilyina E. A., Saraev L. A. Modeling of phase transformations and superelastic hardening of unstable materials, Vestn. Samar. Gos. Tekhn. Univ., Ser. Fiz.-Mat. Nauki [J. Samara State Tech. Univ., Ser. Phys. Math. Sci.], 2018, vol. 22, no. 3, pp. 407-429 (In Russian). https://doi.org/10.14498/vsgtu1626.

7. Drin Ya., Ushenko Yu., Drin I., Drin S. About the approximate solutions to linear and non-linear pseudodifferential reaction diffusion equations, Mohyla Math. J., 2019, vol. 2, pp. 41-45. https://doi.org/10.18523/2617-70802201941-45.

8. Kuttler C. Reaction-diffusion equations with applications, Lecture Notes; Munich Technical University, 2011. https://www-m6.ma.tum.de/ kuttler/script_reaktdiff.pdf.

9. Uvarova L. A., Krivenko I. V., Ivannikov A. F. Peculiarities of stochastic resonance in disperse systems, In: Australian Institute of Physics 17th National Conference 2006: Refereed Papers (3-8 December, 2006; Brisbane, Australia). Canberra, 2006, 274.

10. Uvarova L. A., Babarin S. S. The movement of molecules and nanoparticles in potential field with the Casimir force in nano volumes with different optical boundaries, Physica Scripta, 2014, vol.2014, no.T162, 014053. https://doi.org/10.1088/0031-8949/2014/ T162/014053.

11. Uvarova L. A., Fedyanin V. K. Scattering of an electromagnetic wave by a spherical particle with nonlinear properties, Preprint no. P17-89-372. Dubna, JINR, 1989, 7 pp. (In Russian)

12. Uvarova L. A. Some exact solutions for the vector of electric field strength in conjugate nonlinear media, Preprint no. P17-87-693. Dubna, JINR, 1987, 14 pp. (In Russian)

13. Lakhno V. D., Uvarova L. A. The effect of electron heating of proteins on the electron transport rate, Preprint. Pushchino, Pushchino Scientific Center of RAS, 1993, 13 pp. (In Russian)

14. Chikova O. A. Structural transitions in complexly alloyed melts, Izvestiya. Ferrous Metallurgy, 2020, vol.63, no.3-4, pp. 261-270 (In Russian). https://doi.org/10.17073/ 0368-0797-2020-3-4-261-270. 